\title{
Gingival Depigmentation Using Scalpel Technique: A Case Report
}

\author{
Dr. Binod Adhikari, ${ }^{1}$ Dr. Shaili Pradhan,${ }^{2}$ Dr. Ranjita Shrestha Gorkhali ${ }^{3}$ \\ ${ }^{1}$ Resident, ${ }^{2}$ Professor, ${ }^{3}$ Assistant Professor, Periodontology and Oral Implantology Unit, \\ Department of Dental Surgery, National Academy of Medical Sciences, Bir Hospital, Kathmandu, Nepal
}

\begin{abstract}
Excessive gingival pigmentation is a major esthetic concern for many people. Though, it is not a medical problem, many people complain of dark gums as unaesthetic. Gingival hyperpigmentation is believed to be a genetic trait in populations and is more appropriately termed physiologic or racial gingival pigmentation; a condition, which affects all races differently. Gingival pigmentation can be removed for esthetic reasons using methods like chemical cauterization, bur abrasion, scalpel surgery, cryosurgery, electrosurgery, gingival grafts and laser techniques. A case is reported here where surgical depigmentation was performed.
\end{abstract}

Key Words: Depigmentation, Gingiva, Melanin, Scalpel Surgery

\section{INTRODUCTION}

Physiological pigmentation of the oral mucosa is clinically manifested as multifocal or diffuse melanin pigmentation with variable amount in different ethnic groups. ${ }^{1}$ Melanin, a brown pigment, is the most common cause of endogenous pigmentation of gingiva. ${ }^{2}$ This problem is aggravated in patients with a "gummy smile" or excessive gingival display while smiling. Gingival depigmentation is a periodontal plastic surgical procedure where gingival hyperpigmentation is removed or reduced by various techniques. ${ }^{3}$ The present case report, describes a simple and effective surgical depigmentation technique that does not require sophisticated instruments yet yields esthetically acceptable results along with Patient's satisfaction.

\section{CASE REPORT}

A 34 year old female patient reported to Department of Dental Surgery, Periodontology and Oral Implantology unit, Bir Hospital, with a chief complaint of blackish discoloration of the upper and lower front gingival regions (Figure 1) which she felt as esthetically unappealing. The discoloration was not associated with other symptoms like paresthesia, ulceration or discharge. She was systemically healthy and non-smoker. Her oral examination revealed that she had deeply pigmented gingiva from right first premolar to left first premolar, both

\section{Correspondence:}

Dr. Binod Adhikari

Periodontology and Oral Implantology Unit,

Department of Dental Surgery, National Academy of Medical Sciences, Bir Hospital, Mahabouddha, Kathmandu, Nepal

email:drbinod25@yahoo.com

\section{Citation}

Adhikari B, Pradhan S, Gorkhali RS. Gingival depigmentation using scalpel technique: a case report. J Nep Soc Perio Oral Implantol. 2017;1:40-1. in the upper and lower arches. The patient requested for an esthetic treatment which could make her "black" colored gums look better.

The depigmentation procedure with scalpel was planned accordingly. After the administration of a local anesthetic solution, a Bard Parker handle with a No. 15 blade was used to remove the pigmented layer (Figure 2). Pressure was applied with sterile gauze to control hemorrhage during the procedure. The entire pigmented epithelium along with a thin layer of connective tissue was removed. The exposed surface was irrigated with saline; the surgical area was covered with a periodontal dressing. Post-surgical instructions were given along with antibiotics (Amoxicillin $500 \mathrm{mg}$, three times daily for 5 days) and anti-inflammatory analgesics (Ibuprofen and Paracetamol three times daily for 3 days). The patient was advised to use $0.2 \%$ chlorhexidine gluconate mouth wash 12 hourly for 1 week.
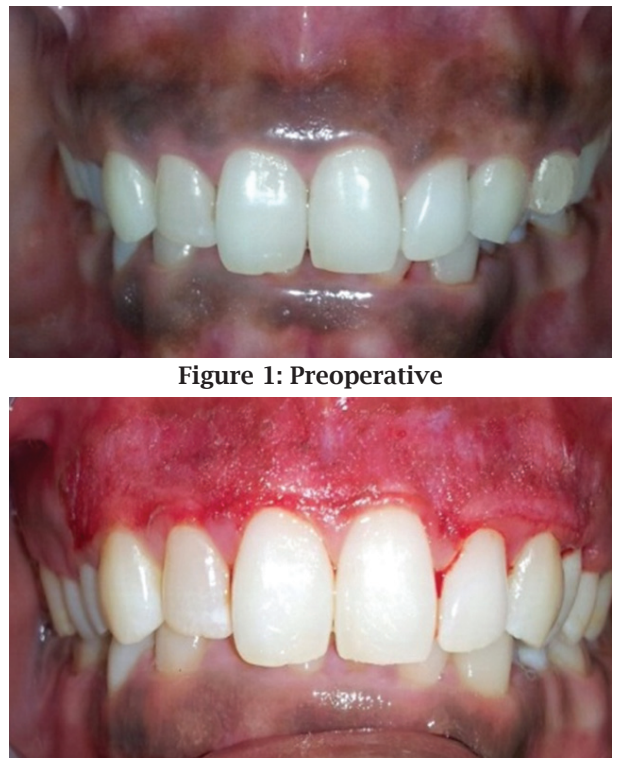

Figure 2: Just after depigmentation 


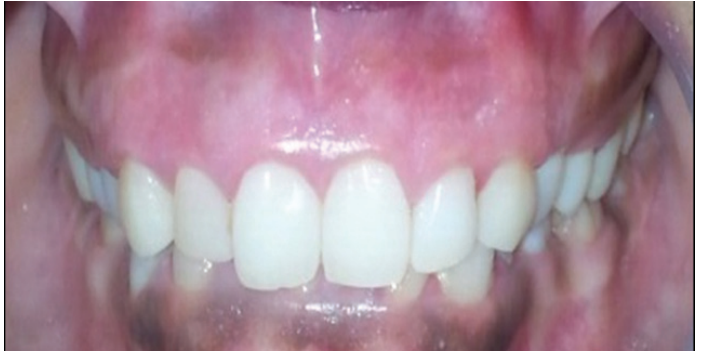

Figure 3: After 9 months

The patient was reviewed at the end of 1 week, then after 1, 3 and 9 months respectively. The healing was uneventful without any post operative pain or sensitivity. The gingiva appeared healthy and no repigmentation was observed at the end of 9 months (Figure 3).

\section{DISCUSSION}

Four factors are responsible for giving color to the gingiva, they are - vascular supply, thickness of the epithelium, degree of keratinization of epithelium, and presence of pigmentcontaining cells. Melanin pigmentation is frequently caused by melanin deposition by active melanocytes located mainly in the basal layer of the oral epithelium. Physiologic pigmentation is probably genetically determined, but as Dummet suggested, the degree of pigmentation is partially related to mechanical, chemical, and physical stimulation.

The foremost indication for depigmentation therapy is the demand by a person for improved esthetics. Various methods as gingivectomy, gingivectomy with free gingival autografting, acellular dermal martrix allografts, electrosurgery, cryosurgery, abrasion with diamond bur, and lasers have been used for cosmetic therapy of gingival melanin depigmentation. ${ }^{3}$ Selection of the technique should be based on clinical experience, patient's affordability and an individual preference of the clinician. ${ }^{4}$

Electrosurgery requires more expertise than scalpel surgery. Prolonged or repeated application of current to tissue induces heat accumulation and undesired tissue destruction. Contact with periosteum or alveolar bone and vital teeth should be avoided. ${ }^{5}$
Cryosurgery is followed by considerable swelling, and increased soft tissue destruction. Depth control is difficult, and optimal duration of freezing is not known, but prolonged freezing increases tissue destruction. ${ }^{6}$ Depigmentation with lasers achieves good results, but they require sophisticated equipment, and are expensive. ${ }^{7}$

A free gingival graft can also be used to eliminate the pigmented areas. However, it requires a donor site and color matching may be a problem. ${ }^{8}$ These treatment modalities, however, are not widely accepted or popularly used.

Scalpel surgical technique is highly recommended in consideration of the equipment avaibility. It is known that the healing period for scalpel wounds are faster than other techniques. However, scalpel surgery may cause unpleasant bleeding during and after the operation, and it is necessary to cover the exposed area with periodontal dressing for 7 to 10 days. ${ }^{9}$ This technique is simple and versatile requiring minimum armamentarium.

Though the initial result of the depigmentation surgery is highly encouraging, repigmentation is a common problem. Repigmentation is described as spontaneous and has been attributed to the activity and migration of melanocytic cells from surrounding areas. The mechanism of repigmentation is not understood completely, but according to the "migration theory," active melanocytes from adjacent pigmented tissues migrate to treated areas causing repigmentation. ${ }^{10}$ Pigment recurrence has been documented to occur, following the surgical procedure, within 24 days to 8 years long period. In the present case, no areas of repigmentation were seen at the end of 9 months. The case is being followed up to assess if there is any recurrence of melanin pigmentation.

\section{CONCLUSION}

In this age of smile-consciousness, there is a growing demand for aesthetic dental treatment. Gingival melanin hyperpigmentation is a commonly encountered aesthetic problem. The surgical depigmentation procedure described in this case report was found to be simple, economical and clinically effective treatment modality for the management of gingival melanin pigmentation leading to an aesthetically pleasing outcome.

\section{REFERENCES}

1. Cicek Y, Ertas U. The normal and pathological pigmentation of oral mucous membrane: a review. J Contemp Dent pract. 2003;4(3):76-86.

2. Dummett CO, Barens G. Oromucosal pigmentation: an updated literary review. J periodontol. 1971;42(11):726-36.

3. Kasagani SK, Nutalapati R, Mutthineni RB. Esthetic depigmentation of anterior gingiva. A case series. The New York state Dent J. 2012;78(3):26-31.

4. Roshna T, Nandakumar K. Anterior esthetic gingival depigmentation and crown lengthening: report of a case. J Contemp Dent Pract. 2005;6(3):139-47.

5. Gnanasekhar JD, al-Duwairi YS. Electrosurgery in dentistry. Quintessence Int. 1998;29(10):649-54.

6. Yeh CJ. Cryosurgical treatment of melanin-pigmented gingiva. Oral Surg, Oral Med, Oral Pathol, Oral Radiol, Endod. 1998;86(6):660-3.

7. Ozbayrak S, Dumlu A, Ercalik-Yalcinkaya S. Treatment of melanin-pigmented gingiva and oral mucosa by CO2 laser. Oral Surg, Oral Med, Oral Pathol, Oral Radiol, Endod. 2000;90(1):14-5.

8. Tamizi M, Taheri M. Treatment of severe physiologic gingival pigmentation with free gingival autograft. Quintessence Int. 1996;27(8):555-8.

9. Almas K, Sadig W. Surgical treatment of melanin-pigmented gingiva; an esthetic approach. Indian J Dent Res. 2002;13(2):70-3.

10. Mokeem, Sameer A. Management of Gingival Hyperpigmentation by Surgical Abrasion : Report of Three Cases. Saudi Dent J. 2006;18(3):162-66. 